\title{
Detection of lung lesions in breath-hold VIBE and free-breathing Spiral VIBE MRI compared to $\mathrm{CT}$
}

Susann-Cathrin Olthof ${ }^{1}$, Christian Reinert ${ }^{1}$, Konstantin Nikolaou' ${ }^{1}$ Christina Pfannenberg ${ }^{1}$, Sergios Gatidis ${ }^{1}$, Thomas Benkert ${ }^{2}$, Thomas Küstner ${ }^{1 *}$ (D) and Patrick Krumm

\begin{abstract}
Background: Detection of pulmonary nodules in MRI requires fast imaging strategies without respiratory motion impairment, such as single-breath-hold Cartesian VIBE. As patients with pulmonary diseases have limited breath-hold capacities, this study investigates the clinical feasibility of non-Cartesian Spiral VIBE under free-breathing compared to $\mathrm{CT}$ as the gold standard.

Methods: Prospective analysis of 27 oncological patients examined in PET/CT and PET/MR. A novel motion-robust 3D ultrashort-echo-time (UTE) MR sequence was evaluated in comparison with CT and conventional breath-hold MR. CT scans were performed under breath-hold in end-expiratory and end-inspiratory position (CT ex, CT in). MR data was acquired with non-contrast-enhanced breath-hold Cartesian VIBE followed by a free-breathing 3D UTE Spiral VIBE. Impact of respiratory motion on pulmonary evaluation was investigated by two readers in Cartesian VIBE, followed by UTE Spiral VIBE and CT ex and the reference standard of CT in. Diagnostic accuracy was calculated, and visual image quality assessed.
\end{abstract}

Results: Higher detection rate and sensitivity of pulmonary nodules in free-breathing UTE Spiral VIBE in comparison with breath-hold Cartesian VIBE were found for lesions > $10 \mathrm{~mm}$ (UTE Spiral VIBE/VIBE/CT ex): 93\%/54\%/100\%; Lesions 5-10 mm: 67\%/25\%/ 92\%; Lesions < $5 \mathrm{~mm}: 11 \% / 11 \% / 78 \%$. Lobe-based analysis revealed sensitivities and specificities of $64 \% / 96 \% / 41 \%$ and $96 \% / 93 \% / 100 \%$ for UTE Spiral VIBENIBE/CT ex.

Conclusion: Free-breathing UTE Spiral VIBE indicates higher sensitivity for detection of pulmonary nodules than breath-hold Cartesian VIBE and is a promising but time-consuming approach. However, sensitivity and specificity of inspiratory CT remain superior in comparison and should be preferred for detection of pulmonary lesions.

Keywords: Spiral VIBE, Ultrashort echo time, MR lung nodule detection, Tomography (X-ray computed)

*Correspondence: thomas.kuestner@med.uni-tuebingen.de

${ }^{1}$ Department of Diagnostic and Interventional Radiology, University Hospital of Tuebingen, Hoppe-Seyler-Straße 3, 72076 Tuebingen,

Germany

Full list of author information is available at the end of the article

\section{Key points}

- Clinical feasibility of a free-breathing 3D UTE Spiral VIBE sequence.

- Superior detection rate for lesions $>10 \mathrm{~mm}$ in Spiral VIBE compared to VIBE.

- Inferior detection rate of Spiral VIBE compared to CT as gold-standard. 


\section{Introduction}

Magnetic resonance imaging (MRI) of the air-filled lungs suffers from motion artifacts due to the cyclic heartbeat and respiration [1]. The low proton density of only approximately $800 \mathrm{~g}$ for both lungs and fast $\mathrm{T} 2 *$ decay of air result in a low lung signal intensity [2] reflected in a low signal-to-noise ratio (SNR) in the resulting image. Furthermore, susceptibility artifacts caused by the interface of different tissue types lead to field inhomogeneities with short $\mathrm{T} 2$ and $\mathrm{T} 2$ * lung signal in the chest [2].

To overcome the two major problems of low SNR and impact of respiratory motion, previous works aimed at providing higher SNR with the ultrashort echo time (UTE) technique for 3D stack-of-radials or 3D radial imaging [3-7] with reported sensitivities of 73-93\% for lung nodule detection in comparison with the gold standard of radiation-dependent computed tomography (CT) imaging [3]. Moreover, echo time reduction was also achieved with central pointwise encoding and stack-of-radials acquisition (PETRA) to evaluate pulmonary pathologies such as nodules, cystic fibrosis and pulmonary embolisms [8]. Respiratory motion was resolved by freezing respiratory motion via fast single [9] or multiple [10] breath-hold acquisitions.

Even imaging under free-breathing was performed using a fast and motion-robust UTE T1-weighted 3D gradient echo sequence with non-Cartesian stack-ofspirals trajectory readout including short echo times to capture fast T2* decay (UTE Spiral VIBE) [11].

The aim of this study was to investigate the diagnostic accuracy for detection of pulmonary nodules with UTE Spiral VIBE sequence acquired under free-breathing conditions in clinical routine without i.v. contrast application. We hypothesized a prolonged scan time of UTE Spiral VIBE may be justified for better lung lesion detectability in comparison with: 1) end-expiratory breath-hold Cartesian VIBE acquired on MRI and baseline reference, 2) CT in inspiration and 3) expiration.

\section{Methods}

Study cohort

Prospective, consecutive analysis of 27 clinically induced $\mathrm{PET} / \mathrm{CT}$ examinations with following, voluntary PET/ MR examination was performed, independent of the pulmonary findings in chest CT. Patients gave written consent, and the local review board approved this prospective study. PET/MR acquisition included VIBE and UTE Spiral VIBE sequences for lung evaluation. Mean patient age was $65.6 \pm 10$ years (range $45-83$ years), 7 females (26\%). Primary diagnosis for $24{ }^{18} \mathrm{~F}$-FDG PET/ CT examinations were lung lesions $(n=16)$, non-Hodgkin lymphoma $(n=3)$, cholangio-cellular carcinoma $(n=1)$, breast-cancer $(n=1)$, melanoma $(n=2)$ and rectum cancer $(n=1)$, as well as neuroendocrine tumors NET ( $n=2,{ }^{68}$ Ga-DOMITATE PET), prostate cancer $\left(n=1{ }^{68}\right.$ Ga-PSMA PET). Exclusion criteria were aged under 18 years and contraindications for MR imaging and pleural effusion.

\section{Data acquisition}

All images were acquired on a $3 \mathrm{~T}$ PET/MR scanner in supine position with both arms besides the body (Biograph mMR, Siemens Healthcare, Erlangen, Germany) and on a PET/CT scanner with elevated arms on the same day, applying standardized examination and acquisitions parameters (Biograph mCT, Siemens Healthcare, Knoxville, TN, USA). Details of the examination protocol and scan parameters are depicted in Table 1 and Fig. 1.

\section{MRI}

3D T1-weighted spoiled gradient echo sequence (VIBE) was acquired in expiration phase in axial plane, with Cartesian trajectory, centric reordering, without parallel imaging. Two echoes were recorded for DIXON-based fat-water separation. Field-of-view placement and orientation were similar to CT. Image acquisition time was $14 \mathrm{~s}$ for breath-hold VIBE. Additionally, a prototypical UTE Spiral VIBE acquisition was performed without i.v. contrast application (as patients already received

Table 1 Overview of the MR and CT data acquisition details

\begin{tabular}{|c|c|c|c|c|c|}
\hline & VIBE & UTE Spiral VIBE & & CT expiration & CT inspiration \\
\hline Resolution [mm $\left.\mathrm{mm}^{3}\right]$ & $1.3 \times 1.3 \times 3$ & $\begin{array}{l}1.5 \times 1.5 \times 1.5 \text { (iso- } \\
\text { tropic resolution) }\end{array}$ & Resolution $\left[\mathrm{mm}^{3}\right]$ & $0.8 \times 0.8 \times 0.7$ & $0.8 \times 0.8 \times 0.7$ \\
\hline Matrix & $320 \times 240$ & $320 \times 320$ & Matrix & $512 \times 512$ & $512 \times 512$ \\
\hline Repetition time [ms] & 3.9 & 3.2 & Peak tube voltage [kV] & 120 & 120 \\
\hline Echo time [ms] & 2.5 & 0.05 & Tube current [mAs] & Dose modulated & Dose modulated \\
\hline Acquisition time [s] & $14 \pm 1$ & $409 \pm 15$ & Convolution kernel & $131 \mathrm{f} / 2$ & $130 f / 2$ \\
\hline Bandwidth [Hz/pixel] & 1185 & 975 & & & \\
\hline Flip angle $\left[^{\circ}\right]$ & 10 & 5 & & & \\
\hline
\end{tabular}




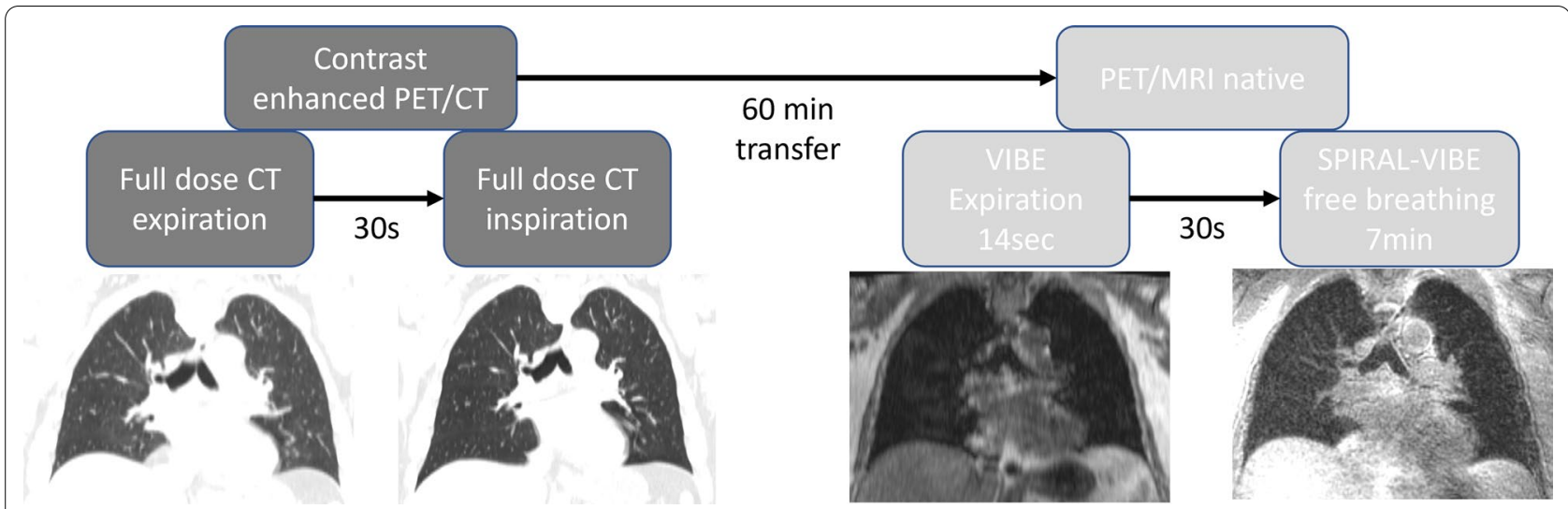

Fig. 1 Flowchart of examination sequence in integrated PET/CT and PET/MRI scanners

CT contrast media prior on the same day), using spiral sampling in the kx-ky plane and Cartesian sampling in $\mathrm{kz}$ direction. By employing short rectangular pulses, a center-out acquisition, and variable TE encoding [12], an echo time of 50 us could be achieved. Therefore, compared with Cartesian VIBE, faster relaxing T2* tissues can be captured. To reduce the number of through-plane phase-encoding steps, images were acquired in coronal orientation. Data acquisition was performed during free-breathing. To avoid corresponding motion artifacts, prospective respiratory gating was performed, for which a self-navigator signal based on intermitted navigator pulses in the SI direction was evaluated during scanning, and scanning was automatically stopped once sufficient data had been acquired in end-expiratory state. Acquisition time therefore depends on the patient's breathing pattern and ranged from 6:30 to7 minutes per patient depending on the acquired slabs.

\section{$C T$}

For reference standard of lung lesion detection and evaluation, chest $\mathrm{CT}$ in inspiration (CT in) was used, which is part of our house-intern standard PET/CT protocol. Additional whole-body CT in expiration (CT ex), clinically necessary for the PET attenuation correction, served as comparative CT scan to the gold standard. Both scans were acquired after i.v. contrast agent application $(120 \mathrm{ml}$, flow rate $2.5 \mathrm{ml} / \mathrm{s}$; Ultravist 370 Bayer Healthcare Pharmaceuticals Berlin, Germany).

\section{Image evaluation}

All data were analyzed in axial plane starting with VIBE, UTE Spiral VIBE, CT in expiration and inspiration using syngo.via Client 5.1 for PET/MRI and PET/CT examinations by two blinded readers with 7 and 9 years of oncological experience including fellowships in nuclear medicine (syngo.MM Oncology VB30A, Siemens Healthcare, Erlangen, Germany; Fig. 1). Calcified lesions and dystelectases were omitted. The longest lesion diameter was measured in lung window settings for CT. If the lesion was not found in inspiratory chest $\mathrm{CT}$, the lesion was defined as 'false positive,' whereas 'false negative' lesions were not detected in any method except inspiratory CT. Pulmonary lobes without any lesions in inspiratory chest CT were counted as 'true negative.' Additionally, image quality was assessed for both CT and MRI imaging applying a four-point scale according to Kumar et al. [13]. Evaluation criteria included the sharpness, potential artifacts, image noise and overall image quality with scores of (1) for perfect image quality, (2) for clinically sufficient image quality, (3) for moderate image quality and (4) for impaired image quality. Lung lesions were analyzed according to their number, their lobe location and their largest size and categorized in smaller than $5 \mathrm{~mm}, 5$ to $10 \mathrm{~mm}$ and over $10 \mathrm{~mm}$.

\section{Statistical analysis}

Categorical, continuous variables and frequencies are given as means with standard deviation (SD). Sensitivity $(\mathrm{Sn})$ and specificity $(\mathrm{Sp})$ were calculated for VIBE, UTE Spiral VIBE and CT ex in comparison with the gold standard of CT in for the whole lung in each patient (lesion-based analysis) and according to the lobe (lobebased analysis). Intraclass correlation coefficients were created for inter-reader agreement for lesion detection in Spiral VIBE. For objective (Spiral) VIBE sequence evaluation, a coefficient of variation (mean and SD) was calculated via SD of the index lesion divided through the mean of the index lesion per patient. Furthermore, the contrast difference ratio (mean and SD) was calculated via contrast-to-noise ratio (contrast between index lesion and lung surrounding) divided through the signal-to-noise 
ratio for each index lesion per patient. All statistical analyses were performed with MedCalc (Version 12.6 MedCalc Software, Ostend, Belgium).

\section{Results}

In 27 patients, 99 lung lesions were detected in total in the inspiration chest $\mathrm{CT}$, which served as gold standard. Lung lesions per patient varied from one to nine, with a mean value of 3.4 per patient (SD 2.6). Size varied from 2 to $122 \mathrm{~mm}$ (mean $13 \mathrm{~mm}$, SD $22 \mathrm{~mm}$ ).

Representative cases of good and difficult pulmonary node detection are given in Figs. 2 and 3.

\section{Lesion-based analysis}

$\mathrm{CT}$ in expiration revealed the highest detection rate for pulmonary lesions in comparison with the reference standard of CT in inspiration (88\%). Accordingly, UTE Spiral VIBE was superior to VIBE for pulmonary lesion detection ( $47 \%$ vs. $26 \%$ ), especially for the detection of lesions between 5 and $10 \mathrm{~mm}$ (67\% vs. 25\%) and lesions over $10 \mathrm{~mm}$ (93\% vs. $54 \%)$. However, no difference was stated between both MR imaging techniques for smaller nodules of $5 \mathrm{~mm}$ (11\%; Table 2).

\section{Lobe-based analysis}

Analysis of the detected lesions per lobe revealed higher detection rate for VIBE (41\%) and UTE Spiral VIBE (64\%) as well as CT in expiration (93\%) in comparison with the gold standard of chest $\mathrm{CT}$ in inspiration. Specificity of both VIBE and Spiral VIBE was $96 \%$ and for chest CT in expiration $100 \%$ (Table 2).

$\mathrm{CT}$ in expiration showed the highest sensitivity for lung nodule detection (98\%) in comparison with the gold standard, independent of the lesion localization. Both MR sequences were impaired for lesions next to the fissura horizontalis and obliqua. However, UTE Spiral VIBE was superior to VIBE with a sensitivity of $46 \%$ vs. $27 \%$ (Table 3).

\section{Image quality assessment}

Image quality was assessed for each imaging modality with a score from 1 for perfect image quality up to a score of 4 for impaired image quality including the evaluation of the overall image quality. The highest image quality was observed in the gold standard of CT in inspiration. Although UTE Spiral VIBE showed lowest numbers for score 1 image quality in general, the clinical applicable image quality of scores 1 and 2

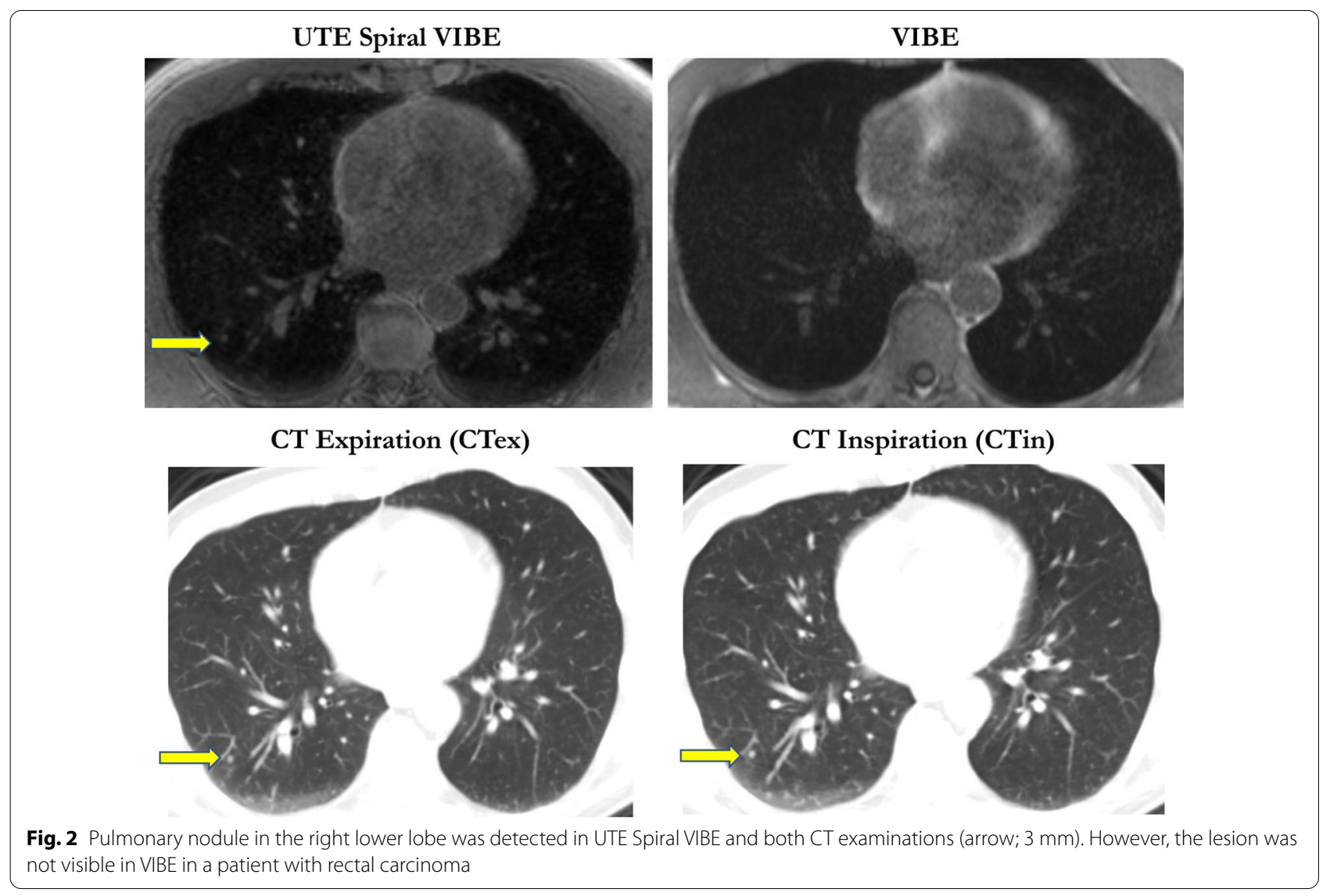




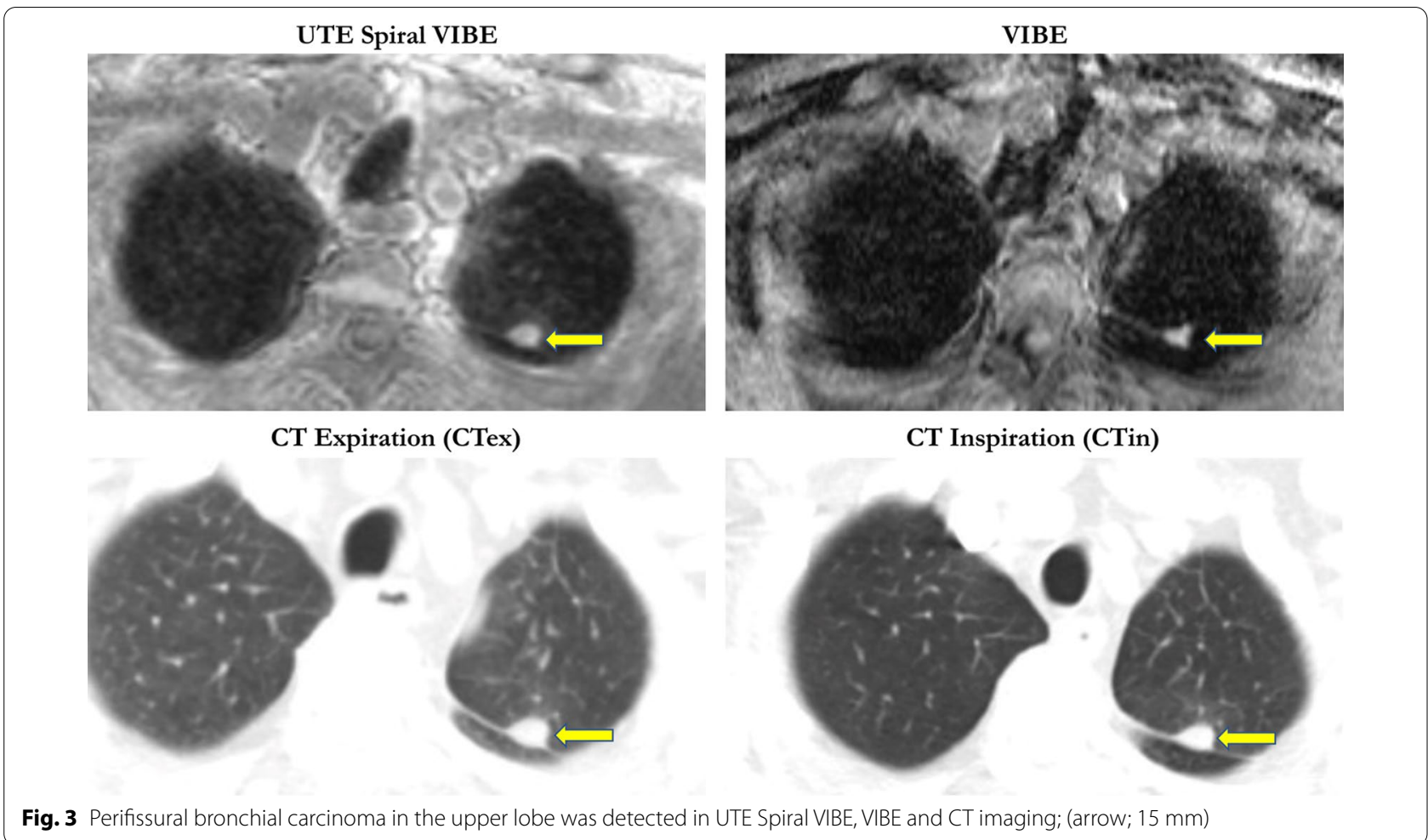

Table 2 Overview of the detected lesions according to MRI sequence VIBE and Spiral VIBE as well as CT in expiration in comparison with the gold standard of CT in inspiration both lesion- and lobe-based

\begin{tabular}{llll}
\hline & VIBE & UTE Spiral VIBE & CT expiration \\
\hline $\begin{array}{l}\text { Detection rate of all lesions } \\
\text { Sensitivity }\end{array}$ & $26 / 99$ & $47 / 99$ & $87 / 99$ \\
$(95 \% \mathrm{Cl})$ & $26 \%$ & $47 \%$ & $88 \%$ \\
Detection rate according to lesion size & $(17-38)$ & $(32-59)$ & $(70-107)$ \\
$<5 \mathrm{~mm}$ & & & \\
& $5 / 47$ & $5 / 47$ & $37 / 47$ \\
$5-10 \mathrm{~mm}$ & $11 \%$ & $11 \%$ & $79 \%$ \\
& $6 / 24$ & $16 / 24$ & $22 / 24$ \\
$>10 \mathrm{~mm}$ & $25 \%$ & $67 \%$ & $92 \%$ \\
& $15 / 28$ & $26 / 28$ & $28 / 28$ \\
Lobe-based analysis & $54 \%$ & $93 \%$ & $100 \%$ \\
Sensitivity & $24 / 58$ & $37 / 58$ & $54 / 58$ \\
$(95 \% \mathrm{Cl})$ & $41 \%$ & $64 \%$ & $93 \%$ \\
Lobe-based analysis & $(41-70)$ & $(26-51)$ & $(41-70)$ \\
Specificity & $74 / 77$ & $74 / 77$ & $77 / 77$ \\
$(95 \% \mathrm{Cl})$ & $96 \%$ & $96 \%$ & $100 \%$ \\
& $(58-93)$ & $(58-94)$ & $(61-96)$ \\
\hline
\end{tabular}

were higher compared to VIBE $(8.5 \%+70.2 \%=78.7 \%$ vs. $23 \%+27 \%=50 \%$; Table 4 ). Mean coefficient of variation was, respectively, $0.170 \pm 0.082$ and $0.112 \pm 0.045$, respectively, for VIBE and Spiral VIBE. 
Table 3 Synopsis of the detected lung lesions according to the anatomical distribution

\begin{tabular}{llll}
\hline $\begin{array}{l}\text { Detection rate according } \\
\text { to localization }\end{array}$ & VIBE & UTE Spiral VIBE & CT expiration \\
\hline Right upper lobe & $9 / 24$ & $11 / 24$ & $21 / 24$ \\
Middle lobe & $0 / 8$ & $1 / 8$ & $8 / 8$ \\
Right inferior lobe & $5 / 21$ & $6 / 21$ & $17 / 21$ \\
Right hilum & $2 / 2$ & $2 / 2$ & $2 / 2$ \\
Left upper lobe & $3 / 19$ & $13 / 19$ & $17 / 19$ \\
Left inferior lobe & $6 / 17$ & $13 / 17$ & $15 / 17$ \\
Left hilum & $1 / 1$ & $1 / 1$ & $1 / 1$ \\
Fissura horizontalis/obliqua & $0 / 7$ & $0 / 7$ & $6 / 7$ \\
Central localization & $23 / 60$ & $38 / 60$ & $70 / 60$ \\
Subpleural localization & $3 / 39$ & $9 / 39$ & $17 / 39$ \\
\hline
\end{tabular}

Table 4 Overview of the image quality for the MRI and CT examination modalities

\begin{tabular}{lllll}
\hline Score & VIBE & UTE Spiral VIBE & CT expiration & CT inspiration \\
\hline 1 & $6 / 26(23 \%)$ & $4 / 47(9 \%)$ & $19 / 87(22 \%)$ & $80 / 99(81 \%)$ \\
2 & $7 / 26(27 \%)$ & $33 / 47(70 \%)$ & $62 / 87(71 \%)$ & $17 / 99(17 \%)$ \\
3 & $13 / 26(50 \%)$ & $10 / 47(21 \%)$ & $6 / 87(7 \%)$ & $2 / 99(2 \%)$ \\
4 & 0 & 0 & 0 & 0 \\
\hline
\end{tabular}

Mean contrast difference ratio was $0.906 \pm 0.028$ and $0.848 \pm 0.061$ for VIBE and Spiral VIBE.

\section{Inter-reader agreement}

The absolute inter-reader agreement for nodule detection was 0.818 (95\% CI 0.7499-0.8697).

\section{Discussion}

This prospective study demonstrates superiority of freebreathing UTE Spiral VIBE in comparison with breathhold VIBE for lung nodule detection in a clinical routine setting at $3 \mathrm{~T}$. For lesions over $10 \mathrm{~mm}$, UTE Spiral VIBE reveals highest detection rates for lung nodules in MRI, comparable to chest $\mathrm{CT}$ in expiration. As a hallmark of this study, both MRI and CT imaging examinations were performed in expiration, resulting in a directly comparable image evaluation without any distortions due to different imaging settings.

Sensitivities for lung nodule detection at $3 \mathrm{~T}$ are with $27 \%$ for VIBE and $48 \%$ for UTE Spiral VIBE in this first readers' experience study inferior to literature data (55\% [14]). This might be caused by the high rate of small lung lesions under $5 \mathrm{~mm}$ in our study in the gold standard of CT imaging (47/99, 48\%). In accordance, current clinical studies [9] and a current animal model indicate MRI suitable for detection of lung lesions larger than $4 \mathrm{~mm}$ [15].
Furthermore, the difference in examination protocols, like the slice thickness and the MR field strength, is crucial. Chassagnon reported significantly lower SNR and contrast-to-noise ratios (CNR) at $1.5 \mathrm{~T}$ in comparison with $3 \mathrm{~T}$ in ten volunteers [16]. Higher SNR is also obtained after i.v. contrast media application [17], explaining the difference in pulmonary detection rates in the literature. Other reasons might be the examination setting, as our patients underwent non-enhanced MR examination directly after PET/CT scan on the same day, in comparison with Ohno, where patients received both examinations within one week [7]. Here, comparable detection rates between $\mathrm{CT}$ with standard and reduced dose as well as UTE were described in 52 patients with similar lung detection diameters to ours (mean 13 vs. $12 \mathrm{~mm}$; SD 22 vs $7.3 \mathrm{~mm}$ [7]). However, the reported high sensitivities of lung nodule detection of $73 \%$ in 82 pulmonary nodules in eight patients examined with UTE [18] are less meaningful without the reported specificities.

With regard to image quality, UTE Spiral VIBE in freebreathing acquisition seems to be more robust for clinical application in comparison with breath-hold VIBE, as the total amount of score- 1 and score- 2 image qualities was higher (78\% vs. 49\%). Improved coefficient of variation with similar contrast ratios for UTE Spiral VIBE in comparison with VIBE supports the observation of a reduced noise floor. Accordingly, UTE Spiral VIBE is less prone to motion artifacts with reported comparable morphological information to CT [19]. Thus, the longer acquisition time of approximately $7 \mathrm{~min}$ for UTE Spiral VIBE in comparison with $14 \mathrm{~s}$ for VIBE seems effective, especially in the whole-body PET/MRI staging for oncological patients with a reduced general and in some cases pulmonary condition. Further potential application for Spiral VIBE might lie in the therapeutic field of radiotherapy planning of the primary lung tumor, if clearly visible, thereby reducing unnecessary breathing stops and further radiation exposure for the patient. However, the main potential role for MR lung examination lies especially in the field of pediatric imaging, to replace low-dose chest CT for lung evaluation. Here, the free-breathing approach can be beneficial especially for age groups that cannot follow breath-hold commands.

Since low-dose and ultra-low-dose CT protocols in inspiration with iterative reconstruction algorithms offer very high sensitivities above $90 \%$ and radiation dose of $0.1 \mathrm{mSv}$, the CT approach also seems a suitable pathway without relevant radiation exposure [20, 21]. The question arises whether it may be more helpful to further improve lung evaluation with low-dose protocols in CT as the diagnostic gold standard, for any MRI approach will have inherent drawbacks due to susceptibility with the organ of interest full of air. A reasonable 
approach for staging MRI may include a lung-specific sequence with the duration of one breath-hold, but it can currently not replace the high sensitivity of a lung CT.

We acknowledge several limitations of this study. The patients' positioning of the arms beneath the body for MR sequences potentially affects the imaging quality of the lung, especially when compared to PET/CT with elevated arms. However, this is a methodological issue, which affects any other comparable study. Furthermore, VIBE sequence was acquired in axial, UTE Spiral VIBE in coronal orientation, impeding the sensitivities for lung nodule detection in VIBE as the respiration occurs in the $z$-axis [10]. Our study was focused on an image-guided detection of lung lesions, without histopathological correlation of the analyzed MR lesions, regarding the clinical relevance. Furthermore, lesions were not analyzed for the morphology (ground glass nodules, part-solid nodules, and solid nodules). This could be crucial for risk assessment of pulmonary nodules in the future.

\section{Conclusion}

Free-breathing UTE Spiral VIBE indicates higher sensitivity for detection of pulmonary nodules than breathhold VIBE and is a promising but time-consuming approach. However, sensitivity and specificity of inspiratory $\mathrm{CT}$ remains superior in comparison and should be preferred for detection of pulmonary lesions in adults. However, we see a potential role for UTE Spiral VIBE for imaging in dose-restricted cohorts, e.g., pediatric patients.

\begin{abstract}
Abbreviations
${ }^{18} \mathrm{~F}-\mathrm{FDG}$ PET/CT: ${ }^{18}$ Fluorine fluorodeoxyglucose positron-emission tomography/computed tomography; CAIPIRINHA: Controlled aliasing in parallel imaging results in higher acceleration; CNR: Contrast-to-noise ratio; CT: Computed tomography; EX: Expiration; FOV: Field of view; GRE sequence: Gradient echo sequence; INS: Inspiration; MRI: Magnetic resonance imaging; NET: Neuroendocrine tumor; PETRA: Pointwise encoding and stack-of-radial acquisition; PSMA: Prostate-specific antigen; SE sequence: Spin echo sequence; SNR: Signal-to-noise ratio; T: Tesla; TE: Echo time; TR: Repetition time; UTE: Ultrashort echo time; VIBE: Volumetric interpolated breath-hold examination.
\end{abstract}

\section{Authors' contributions}

All authors substantially contributed to the content of the manuscript, either via analysis of patient data, preparation and correction of the manuscript. All authors approved this version and have agreed to be personally accountable for the author's own contributions and to ensure that questions related to the accuracy of any part of the work. All authors read and approved the final manuscript.

\section{Funding}

Open Access funding enabled and organized by Projekt DEAL.

\section{Availability of data and materials}

The datasets used during the current study are available from the corresponding author on reasonable request.

\section{Declarations}

Ethics approval and consent to participate

Patients gave written consent, and the local review board approved this prospective study.

\section{Consent for publication}

Not applicable.

\section{Competing interests}

The authors declare that they have no competing interests.

\section{Author details}

${ }^{1}$ Department of Diagnostic and Interventional Radiology, University Hospital of Tuebingen, Hoppe-Seyler-Straße 3, 72076 Tuebingen, Germany. ${ }^{2}$ MR Applications Predevelopment, Siemens Healthcare GmbH, Allee am Roethelheimpark 2, 91052 Erlangen, Germany.

Received: 1 July 2021 Accepted: 6 November 2021

Published online: 24 November 2021

\section{References}

1. Raptis CA, Ludwig DR, Hammer MM, et al. (2019) Building blocks for thoracic MRI: Challenges, sequences, and protocol design. J Magn Reson Imaging 50(3):682-701

2. Puderbach M, Hintze C, Ley S, Eichinger M, Kauczor HU, Biederer J (2007) MR imaging of the chest: a practical approach at 1.5T. Eur J Radiol 64(3):345-355

3. Johnson KM, Fain SB, Schiebler ML, Nagle S (2013) Optimized 3D ultrashort echo time pulmonary MRI. Magn Reson Med 70(5):1241-1250

4. Ohno Y, Koyama H, Yoshikawa T, et al. (2016) Pulmonary high-resolution ultrashort TE MR imaging: comparison with thin-section standard-and low-dose computed tomography for the assessment of pulmonary parenchyma diseases. J Magn Reson Imaging 43(2):512-532

5. Gai ND, Malayeri A, Agarwal H, Evers R, Bluemke D (2016) Evaluation of optimized breath-hold and free-breathing 3D ultrashort echo time contrast agent-free MRI of the human lung. J Magn Reson Imaging 43(5):1230-1238

6. Bannas P, Bell LC, Johnson KM, et al. (2016) Pulmonary embolism detection with three-dimensional ultrashort echo time MR imaging: experimental study in Canines. Radiology 278(2):413-421

7. Ohno Y, Koyama H, Yoshikawa T, et al. (2017) Standard-, reduced-, and no-dose thin-section radiologic examinations: comparison of capability for nodule detection and nodule type assessment in patients suspected of having pulmonary nodules. Radiology 284(2):562-573

8. Dournes G, Grodzki D, Macey J, et al. (2015) Quiet submillimeter MR imaging of the lung is feasible with a PETRA sequence at 1.5 T. Radiology 276(1):258-265

9. Yu N, Yang C, Ma G, et al. (2020) Feasibility of pulmonary MRI for nodule detection in comparison to computed tomography. BMC Med Imaging 20(1):53

10. Both M, Schultze J, Reuter M, et al. (2005) Fast T1- and T2-weighted pulmonary MR-imaging in patients with bronchial carcinoma. Eur J Radiol 53(3):478-488

11. Cha MJ, Park HJ, Paek MY, et al. (2018) Free-breathing ultrashort echo time lung magnetic resonance imaging using stack-of-spirals acquisition: a feasibility study in oncology patients. Magn Reson Imaging 51:137-143

12. Qian Y, Boada FE (2008) Acquisition-weighted stack of spirals for fast highresolution three-dimensional ultra-short echo time MR imaging. Magn Reson Med 60(1):135-145

13. Kumar S, Rai R, Stemmer A, et al. (2017) Feasibility of free breathing Lung MRI for Radiotherapy using non-Cartesian k-space acquisition schemes. Br J Radiol 90(1080):20170037

14. Schwenzer NF, Seith F, Gatidis S, et al. (2016) Diagnosing lung nodules on oncologic MR/PET imaging: comparison of fast T1-weighted sequences and influence of image acquisition in inspiration and expiration breathhold. Korean J Radiol 17(5):684-694 
15. Bonert M, Schneider M, Solyanik O, et al. (2020) Diagnostic accuracy of magnetic resonance imaging for the detection of pulmonary nodules simulated in a dedicated porcine chest phantom. PLoS One 15(12):e0244382

16. Chassagnon G, Martin C, Ben Hassen W, et al. (2019) High-resolution lung MRI with Ultrashort-TE: 15 or 3 Tesla? Magn Reson Imaging 61:97-103

17. Wielputz M, Kauczor HU (2012) MRI of the lung: state of the art. Diagn Interv Radiol 18(4):344-353

18. Burris NS, Johnson KM, Larson PE, et al. (2016) Detection of small pulmonary nodules with ultrashort echo time sequences in oncology patients by using a PET/MR system. Radiology 278(1):239-246

19. Dournes G, Yazbek J, Benhassen W, et al. (2018) 3D ultrashort echo time MRI of the lung using stack-of-spirals and spherical k-Space coverages: evaluation in healthy volunteers and parenchymal diseases. J Magn Reson Imaging 48(6):1489-1497

20. Sui $X$, Meinel FG, Song W, et al. (2016) Detection and size measurements of pulmonary nodules in ultra-low-dose CT with iterative reconstruction compared to low dose CT. Eur J Radiol 85(3):564-570

21. Liang T, Du Y, Guo C, et al. (2021) Ultra-low-dose CT-guided lung biopsy in clinic: radiation dose, accuracy, image quality, and complication rate. Acta Radiol 62(2):198-205

\section{Publisher's Note}

Springer Nature remains neutral with regard to jurisdictional claims in published maps and institutional affiliations.

\section{Submit your manuscript to a SpringerOpen ${ }^{\circ}$ journal and benefit from:}

- Convenient online submission

- Rigorous peer review

- Open access: articles freely available online

- High visibility within the field

Retaining the copyright to your article

Submit your next manuscript at $\boldsymbol{\nabla}$ springeropen.com 